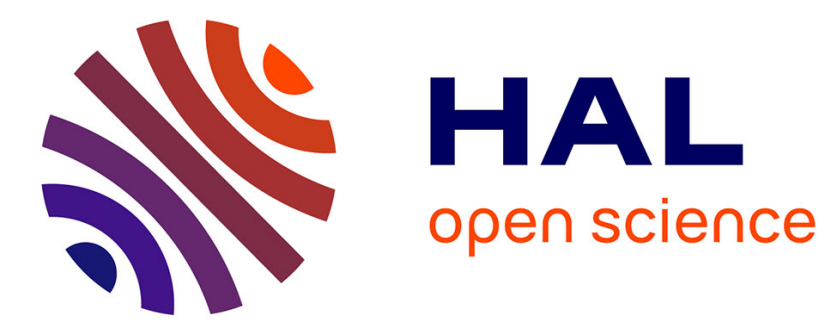

\title{
Propriétés optiques de MnAu2
}

Ch. Jung, M. Ph. Stoll

\section{- To cite this version:}

Ch. Jung, M. Ph. Stoll. Propriétés optiques de MnAu2. Journal de Physique Lettres, 1977, 38 (13), pp.259-262. 10.1051/jphyslet:019770038013025900 . jpa-00231372

\section{HAL Id: jpa-00231372 https://hal.science/jpa-00231372}

Submitted on 1 Jan 1977

HAL is a multi-disciplinary open access archive for the deposit and dissemination of scientific research documents, whether they are published or not. The documents may come from teaching and research institutions in France or abroad, or from public or private research centers.
L'archive ouverte pluridisciplinaire HAL, est destinée au dépôt et à la diffusion de documents scientifiques de niveau recherche, publiés ou non, émanant des établissements d'enseignement et de recherche français ou étrangers, des laboratoires publics ou privés. 


\title{
PROPRIÉTÉS OPTIQUES DE $\mathrm{MnAu}_{2}$
}

\author{
Ch. JUNG et M. Ph. STOLL \\ Laboratoire Pierre-Weiss $\left(^{*}\right)$, 5, rue de l'Université, 67084 Strasbourg, France
}

(Reçu le 25 mars 1977, accepté le 3 juin 1977)

\begin{abstract}
Résumé. - On a déterminé par une méthode polarimétrique les constantes optiques de $\mathrm{MnAu}_{2}$ entre 0,5 et 3,5 eV. L'absorption intrabande est dominante en-dessous de $1 \mathrm{eV}$; avec $m^{*}=2,06 m_{0}$ et $\tau=2,4 \times 10^{-15} \mathrm{~s}$. Le processus interbandes se manifeste à partir de $1 \mathrm{eV}$ et on a mis en évidence un seuil parabolique à 2,17 eV qu'on attribue aux transitions issues du sommet de la bande (d) de l'or vers le niveau de Fermi. Les états (d) occupés du manganèse seraient situés autour de $1 \mathrm{eV}$. La masse effective élevée suggère la présence d'une sous-bande (d) du manganèse autour de $E_{\mathrm{F}}$.
\end{abstract}

Abstract. - By means of a polarimetric method, the optical constants of $\mathrm{MnAu}_{2}$ between 0.5 and $3.5 \mathrm{eV}$ were determined. Intraband absorption is dominant below $1 \mathrm{eV}$; with $m^{*}=2.06 m_{0}$ and $\tau=2.4 \times 10^{-15} \mathrm{~s}$. Interband processes become evident from $1 \mathrm{eV}$ upwards and a parabolic edge, observed at $2.17 \mathrm{eV}$, is attributed to transitions from the top of the gold $\mathrm{d}$ band toward the Fermi level. The occupied states of the manganese are thought to be situated around $1 \mathrm{eV}$. The high effective mass suggests the presence of a manganese d sub-band around $E_{\mathrm{F}}$.

1. Introduction. - Le composé défini $\mathrm{MnAu}_{2}$ a été étudié du point de vue de ses propriétés magnétiques par plusieurs auteurs $[1,2,3]$. Sa structure cristalline est quadratique centrée et sa structure magnétique est hélicoïdale. Il présente en particulier le caractère remarquable d'avoir les propriétés d'un antiferromagnétique dans les champs inférieurs à $10 \mathrm{kOe}$ et celles d'un ferromagnétique au-dessus de ce champ seuil. Au-dessus d'une température de $90^{\circ} \mathrm{C} \mathrm{MnAu}_{2}$ est paramagnétique. Il était intéressant d'entreprendre l'étude des propriétés optiques de ce composé en vue d'apporter une contribution à la connaissance de sa structure électronique.

2. Méthode expérimentale et échantillon. - Pour la détermination des constantes optiques, une technique polarimétrique décrite en détail dans un précédent article [4] a été utilisée. Les paramètres ellipsométriques sont enregistrés en fonction de la longueur d'ondes dans un intervalle allant de $0,35 \mu$ à $2,60 \mu$.

L'échantillon est une plaquette polycristalline préparée par A. J. P. Meyer [1]. Il a été poli mécaniquement à la main, d'abord à la pâte à diamant et ensuite à l'alumine (grosseur des grains au stade final : $0,1 \mu$ ).

3. Résultats expérimentaux. - Les résultats présentés ici sont déduits d'expériences effectuées à la température de $295 \mathrm{~K}$ et à la pression atmosphérique.

(*) E.R.A. 464.
Sur la figure 1 on a représenté $\varepsilon_{2} / \lambda$ en fonction de l'énergie $\hbar \omega$ pour $\mathrm{MnAu}_{2}$ (trait plein). Entre $0,49 \mathrm{eV}$ et $1 \mathrm{eV}$ on observe une décroissance rapide et régulière; entre $1 \mathrm{eV}$ et $2,5 \mathrm{eV}$ l'absorption passe par un minimum (autour de $2 \mathrm{eV}$ ) et présente des changements de pente assez nets; à partir de $2,5 \mathrm{eV}$ on observe une croissance marquée et régulière avec néanmoins un léger minimum à $2,93 \mathrm{eV}$.

Si on compare ce tracé avec celui de l'or également représenté sur la figure 1 (en pointillés, d'après des valeurs extraites de la littérature [5]), on remarque que l'absorption de $\mathrm{MnAu}_{2}$ se situe nettement au-dessus de celle de l'or du côté des faibles énergies ; en particulier le minimum de $\varepsilon_{2} / \lambda$ est de $10 \mu^{-1}$ pour $\mathrm{MnAu}_{2}$ alors qu'il est de $2 \mu^{-1}$ pour l'or. Du côté des énergies plus élevées les deux comportements tendent à se rapprocher et à $3,25 \mathrm{eV}$ les valeurs de $\varepsilon_{2} / \lambda$ pour les deux substances sont égales.

4. Analyse des résultats. - On a essayé d'analyser le spectre d'absorption de $\mathrm{MnAu}_{2}$, d'une part en termes de transitions intrabandes aux faibles énergies par l'utilisation d'un modèle de Drude; d'autre part en termes de transitions interbandes, en particulier au-dessus de $2 \mathrm{eV}$ environ comme le suggère la similitude des deux tracés de la figure 1. On sait en effet que pour l'or, l'absorption s'interprète bien en termes de transitions intrabandes en dessous de $2 \mathrm{eV}$ environ et de transitions interbandes à partir d'un seuil situé à $2,45 \mathrm{eV}[5,6,7]$. 


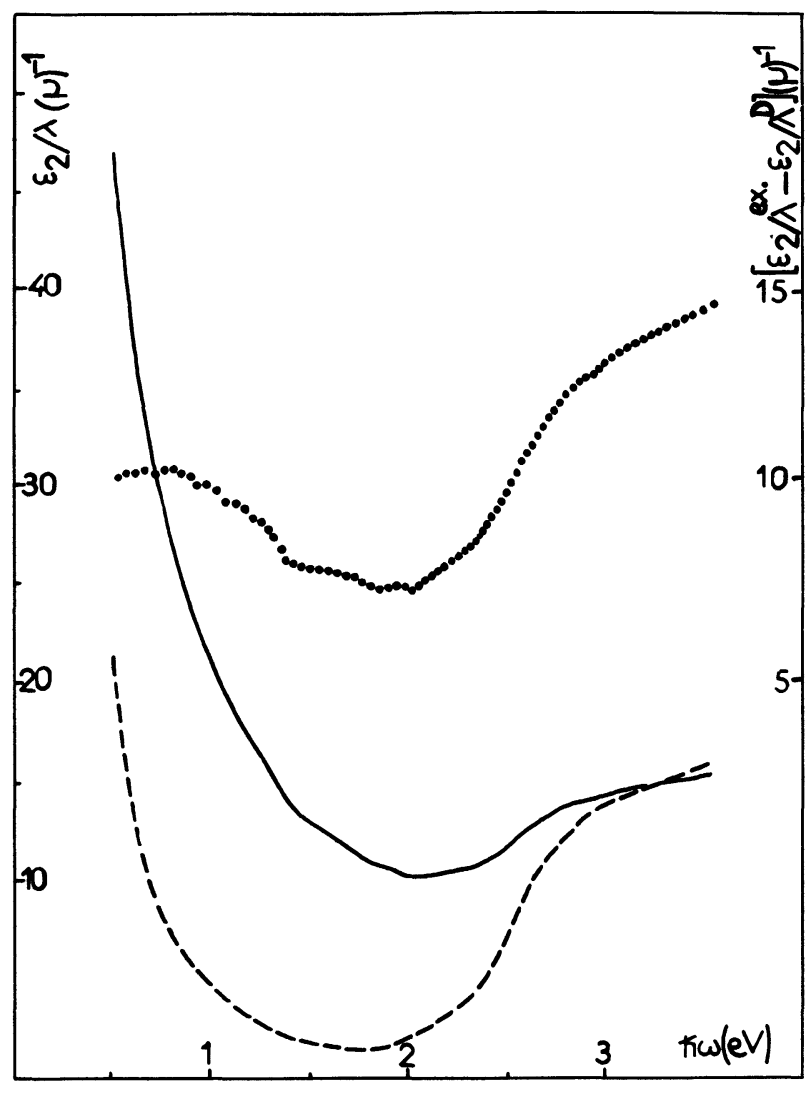

FIG. 1. $-\varepsilon_{2} / \lambda$ en fonction de $\hbar \omega$. En trait plein : présent travail pour $\mathrm{MnAu}_{2}$. En pointillés : pour Au [5]. $\left(\varepsilon_{2} / \lambda\right)_{\exp }-\left(\varepsilon_{2} / \lambda\right)_{\text {Drude }}$ en fonction de $\hbar \omega$ (courbe du haut) (0).

$\left[\varepsilon_{2} / \lambda\right.$ versus $\hbar \omega$. Solid line : present work for $\mathrm{MnAu}_{2}$. Dashed line : for $\mathrm{Au}[5] .\left(\varepsilon_{2} / \lambda\right)_{\exp }-\left(\varepsilon_{2} / \lambda\right)_{\text {Drude }}$ versus $\hbar \omega$ (upper curve) (๑).]

4.1 AbSORPTION INTRABANDE. - On rappelle que les parties réelle et imaginaire $\varepsilon_{1}(\omega)$ et $\varepsilon_{2}(\omega)$ de la constante diélectrique complexe sont données dans le modèle de Drude par :

$$
\begin{aligned}
& \varepsilon_{1}(\omega)=n^{2}-k^{2}=1-\frac{\omega_{\mathrm{p}}^{2}}{\omega^{2}+1 / \tau^{2}} \\
& \varepsilon_{2}(\omega)=2 n k=\frac{1}{\omega \tau} \cdot \frac{\omega_{\mathrm{p}}^{2}}{\omega^{2}+1 / \tau^{2}}
\end{aligned}
$$

avec

$$
\omega_{\mathrm{p}}^{2}=\frac{N e^{2}}{m^{*} \varepsilon_{0}}
$$

où $N$ est le nombre d'électrons de conduction par unité de volume et $m^{*}$ la masse effective de l'électron.

Pour tester la validité du modèle de Drude du côté des faibles énergies, on a représenté sur la figure 2 : $\left(1-\varepsilon_{1}\right)^{-1}$ en fonction de $(\hbar \omega)^{2}$ (diagramme 1$)$ et $\left(1-\varepsilon_{1}\right)$ en fonction de $\varepsilon_{2} / \lambda$ (diagramme 2 , dit d'Argand).

En effet, on constate une très bonne linéarité de ces deux diagrammes en-dessous de $1,025 \mathrm{eV}$. Une méthode de moindres carrés a été employée pour la détermination des paramètres de ces deux droites; ceux-ci permettent de calculer la fréquence de plasma

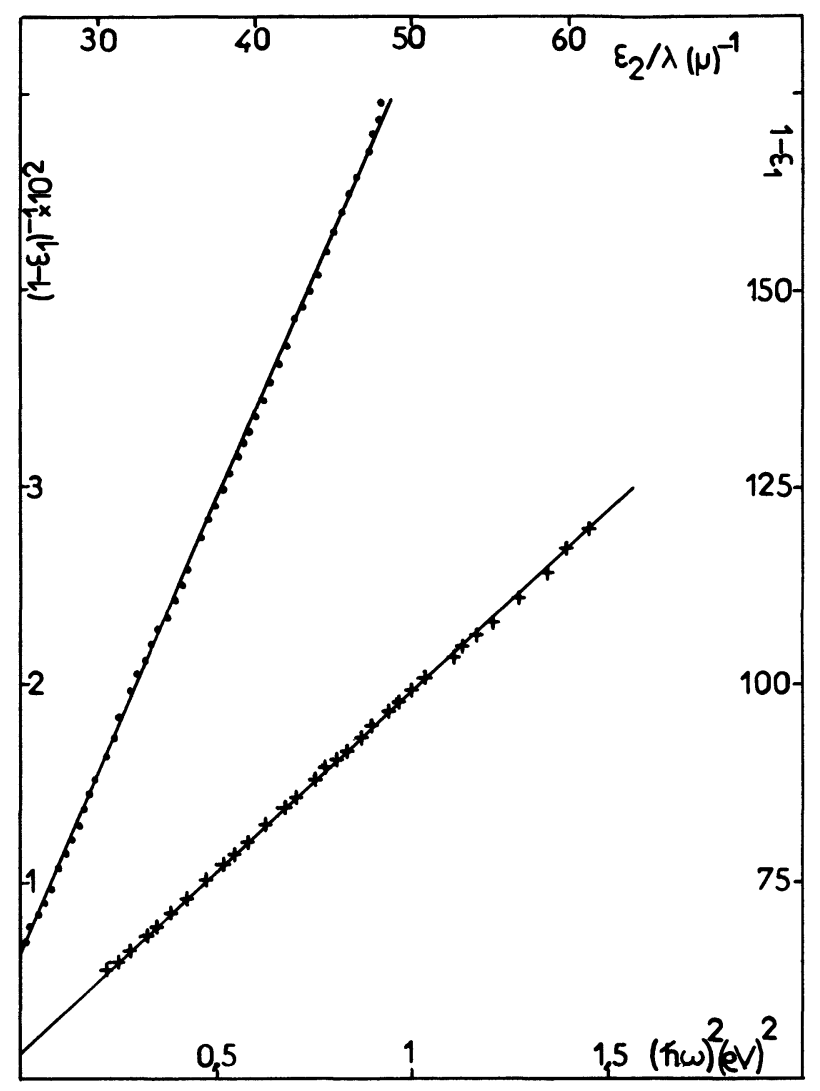

Fig. 2. - Diagramme 1: $\left(1-\varepsilon_{1}\right)^{-1}$ en fonction de $(\hbar \omega)^{2}(+)$. Diagramme 2: $\left(1-\varepsilon_{1}\right)$ en fonction de $\varepsilon_{2} / \lambda(\bullet)$. Le nombre de points marqués sur la figure est inférieur au nombre de points relevés sur les enregistrements et utilisés pour le calcul par moindres carrés des paramètres. Pour le diagramme 2 (Argand), les origines des axes sont décalées.

[Diagram 1: $\left(1-\varepsilon_{1}\right)^{-1}$ versus $(\hbar \omega)^{2}(+)$. Diagram $2:\left(1-\varepsilon_{1}\right)$ versus $\varepsilon_{2} / \lambda(\bullet)$. The number of dots marked on the figure is less than the number of points taken from the graphs which were used to calculate the parameters by least-squares fits. For diagram 2 (Argand) the origins of the axes are shifted.]

$\omega_{\mathrm{p}}$ ainsi que le temps de relaxation $\tau$ pour le diagramme 1 , et le temps de relaxation seul pour le diagramme d'Argand.

Voici les résultats obtenus à partir du diagramme 1 : $\omega_{\mathbf{p}}=1,123 \times 10^{16}\left(\mathrm{~s}^{-1}\right)$ et $\tau=2,371 \times 10^{-15} \mathrm{~s}$ et à partir du diagramme $2: \tau=2,425 \times 10^{-15} \mathrm{~s}$. On remarque que les deux déterminations de $\tau$ correspondent à $2 \%$ près.

Pour calculer la masse effective $m^{*}=\varepsilon_{0} \omega_{\mathrm{p}} / N e^{2}$, il est nécessaire de connaître la valeur de $N$. Sachant que la maille élémentaire, en accord avec la masse volumique [2], comporte 4 atomes d'or et 2 atomes de manganèse, nous avons fait l'hypothèse que chaque atome d'or contribue pour un électron et que chaque atome de manganèse contribue pour deux électrons à l'absorption intrabande; il y a donc un total de 8 électrons de conduction par maille élémentaire. On en déduit $N=8,15 \times 10^{28}$ électrons (s) par $\mathrm{m}^{3}$ la masse effective $m^{*}=2,06 m_{0}\left(m_{0}\right.$ est la masse de l'électron libre). 
En comparant les valeurs trouvées ici pour $\omega_{\mathrm{p}}$, $\tau$ et $m^{*} / m_{0}$ avec les valeurs correspondantes obtenues pour l'or, on remarque que les rapports varient, selon les auteurs $[5,6,7]$, de 0,80 à 0,86 pour $\omega_{p} /\left(\omega_{p}\right)_{A u}$; de 0,24 à 0,39 pour $\tau /(\tau)_{\text {Au }}$ et de 1,86 à 2,19 pour $m^{*} /\left(m^{*}\right)_{\mathrm{Au}}$.

En résumé, on constate que pour $\mathrm{MnAu}_{2}$ le temps de relaxation est en moyenne 2,5 à 4 fois plus petit que celui de l'or et que la masse effective est environ 2 fois plus grande que celle de l'or.

On note aussi que contrairement à ce qui se passe pour l'or, $\varepsilon_{2} / \lambda$ ne varie pas linéairement en fonction de $\lambda^{2}$ pour $\mathrm{MnAu}_{2}$ : ceci provient du fait que $1 / \tau^{2}$ n'est pas négligeable avant $\omega^{2}$ dans l'expression de $\varepsilon_{2}(\omega)$. De plus dans cette représentation on observe que $\left(\varepsilon_{2} / \lambda\right)$ ne passe pas par l'origine, mais présente un décalage sensiblement constant d'environ $10 \mu^{-1}$ par rapport à la courbe calculée à partir des paramètres de Drude et ceci dans tout le domaine situé en-dessous de $1 \mathrm{eV}$.

4.2 ABSORPTION INTERBANDES. - L'absorption intrabande de type Drude a été bien mise en évidence en dessous de $1 \mathrm{eV}$. Habituellement, la contribution interbande s'obtient en soustrayant aux valeurs expérimentales les valeurs calculées à partir des paramètres de Drude. La différence $\left(\varepsilon_{2} / \lambda\right)_{\exp }-\left(\varepsilon_{2} / \lambda\right)_{\text {Drude }}$ en fonction de $\hbar \omega$ a été représentée sur la figure 1 (courbe $\mathrm{du}$ haut). On constate d'abord, dans le domaine Drude en dessous de $1 \mathrm{eV}$, que l'absorption résiduelle n'est pas nulle, ce qui signifie que la contribution intrabande calculée à partir des paramètres $\omega_{p}$ et $\tau$ ne rend pas compte quantitativement de l'absorption expérimentale. Comme on vient de le dire à la fin du paragraphe 4.1 , cette contribution résiduelle à l'absorption est sensiblement constante en-dessous de $1 \mathrm{eV}$ et de l'ordre de $10 \mu^{-1}$, néanmoins elle s'amenuise progressivement aux énergies supérieures à $1 \mathrm{eV}$ alors qu'apparaissent des contributions nouvelles qui se manifestent par des changements de pente très nets à $1,4 \mathrm{eV}, 1,8 \mathrm{eV}$ et $2,35 \mathrm{eV}$. A partir de $2 \mathrm{eV}$ on constate une augmentation franche de l'absorption : un phénomène de ce genre traduit généralement l'apparition d'une contribution interbande importante. Dans le cas de l'or, l'augmentation rapide de l'absorption dans cette région correspond aux transitions issues de la bande (d) vers le niveau de Fermi ; cette absorption se caractérise par un profil parabolique de la quantité $\varepsilon_{2}^{\text {inter }} \times(\hbar \omega)^{2}$ en fonction de $\hbar \omega$.

Etant donnée la similitude entre l'absorption de l'or et celle de $\mathrm{MnAu}_{2}$, il est intéressant d'essayer d'analyser le profil de l'absorption de ce composé au-dessus de $2 \mathrm{eV}$. La question se pose de savoir si le terme de Drude calculé représente bien la contribution intrabande, puisqu'on constate un écart important entre celui-ci et l'absorption expérimentale aux faibles énergies. Le même problème existe d'ailleurs pour l'or, où certains auteurs ont également observé un terme constant, mais relativement faible. On ne voit pas pourquoi cette contribution résiduelle qui ne peut être caractérisée qu'aux faibles énergies et dont l'origine n'apparaît pas clairement, devrait être prise en compte telle quelle dans un domaine d'énergies élevées.

Pour cette raison on a représenté sur la figure 3 $\left[\left(\varepsilon_{2} / \lambda^{2}\right)_{\exp }-\left(\varepsilon_{2} / \lambda^{2}\right)_{\text {Drude }}\right]^{2}$ en fonction de $\hbar \omega$ de $2 \mathrm{eV}$ à $3 \mathrm{eV}$. On observe une zone parfaitement linéaire entre 2,5 et $2,85 \mathrm{eV}$, ce qui pourrait indiquer qu'il existe ici une contribution interbande de même type que pour l'or. La droite des moindres carrés (définie à mieux que $0,3 \%$ ) coupe l'axe des abscisses à $2,17 \mathrm{eV}$, ce qui est une manière de définir la position du seuil de cette absorption interbande.

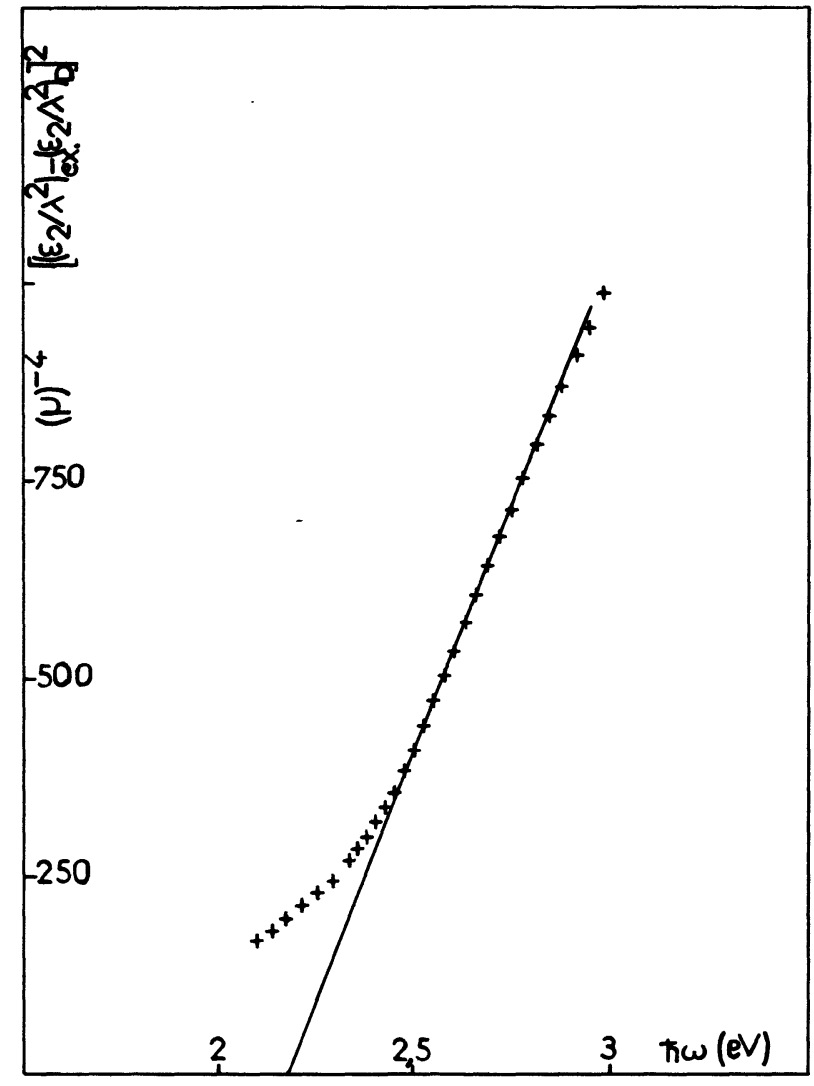

Fig. 3. - Détermination du seuil d'absorption interbande à $2,17 \mathrm{eV}$ $\left[\left(\varepsilon_{2} / \lambda^{2}\right)_{\exp }-\left(\varepsilon_{2} / \lambda^{2}\right)_{\text {Drude }}\right]^{2}$ en fonction de $\hbar \omega$.

[Determination of the interband absorption edge at $2.17 \mathrm{eV}$. $\left[\left(\varepsilon_{2} / \lambda^{2}\right)_{\exp }-\left(\varepsilon_{2} / \lambda^{2}\right)_{\text {Drude }}\right]^{2}$ versus $\left.\hbar \omega.\right]$

5. Discussion et conclusions. - En-dessous de $1 \mathrm{eV}$ le processus intrabande paraît dominant; le modèle de Drude, qui rend compte particulièrement bien de la partie réelle $\varepsilon_{1}$ de la constante diélectrique (cf. diagramme 1 de la figure 2), est caractérisé par une masse effective élevée et un temps de relaxation court. Ces deux aspects ne sont pas contradictoires et indiquent que les porteurs de charges qui contribuent à l'absorption intrabande dans $\mathrm{MnAu}_{2}$ sont moins libres que dans l'or pur; il est donc vraisemblable que dans $\mathrm{MnAu}_{2}$ les états électroniques au voisinage du niveau de Fermi ne sont pas purement (s). 
Plusieurs aspects des propriétés optiques suggèrent que les états $(\mathrm{d})$ du manganèse sont proches du niveau de Fermi :

a) une valeur élevée de $m^{*}$,

b) la droite d'Argand $\left(1-\varepsilon_{1}\right)$ en fonction de $\varepsilon_{2} / \lambda$ (Fig. 2) ne passe pas par l'origine ; son ordonnée à l'origine $\mathbf{P}$ est égale à - 48 comparée à environ - 6 pour l'or [6]. On interprète habituellement ce décalage en écrivant $\varepsilon_{1}(\omega)=\varepsilon_{1}^{\text {Drude }}(\omega)+P$ où $P$ représente la contribution constante à $\varepsilon_{1}$, provenant des transitions interbandes, aux énergies inférieures au seuil d'absorption interbande,

c) l'écart par rapport au modèle de Drude est net au-dessus de $1 \mathrm{eV}$ et on voit apparaître des contributions supplémentaires à l'absorption (cf. Fig. 1).

La possibilité de transitions interbandes à des énergies relativement faibles existe donc, ce qui rend difficile la séparation des contributions intra et interbandes.

En dessous de $1 \mathrm{eV}$, le terme constant qui apparaît dans $\varepsilon_{2} / \lambda$ est très difficile à interpréter. Un tel terme pourrait être le fait d'une contribution interbandes à des énergies faibles, ou encore celui d'une contribution intrabande associée à des porteurs de charges ayant un temps de relaxation très court. Pour l'or, certains auteurs [6] relient la valeur de ce terme à l'état de cristallisation de l'échantillon.

Enfin, au paragraphe 4.2 , on a mis en évidence une contribution interbandes à profil parabolique dont le seuil est situé à $2,17 \mathrm{eV}$. Nous suggérons que cette absorption provient des transitions issues de la bande formée par les états (d) de l'or, dans le composé $\mathrm{MnAu}_{2}$, vers le niveau de Fermi. Le sommet de cette bande serait à environ $2,2 \mathrm{eV}$ au-dessous de ce niveau, comparé à 2,45 eV dans l'or pur.

En résumé, l'esquisse de la structure électronique de $\mathrm{MnAu}_{2}$ pourrait être la suivante : (i) les états (d) de l'or forment une bande identifiable dont le sommet se situe à environ - 2,2 eV par rapport au niveau de Fermi ; (ii) les états (d) du manganèse seraient situés au-dessus de cette bande et groupés autour de $-1 \mathrm{eV}$, dans la mesure où $1 \mathrm{eV}$ apparaît expérimentalement comme une région charnière ; (iii) la présence d'états liés au voisinage du niveau de Fermi suggère que les états (d) du manganèse pourraient être découplés, l'une des sous-bandes chevauchant le niveau de Fermi. Le modèle qui se dégage de l'étude des propriétés optiques n'est pas incompatible avec celui qui a été proposé à partir de mesures de chaleurs spécifiques $[8,9]$.

Une étude expérimentale détaillée, actuellement en cours, en particulier en fonction de la température, devrait permettre de mieux discerner la part prise dans l'absorption par les deux types de transitions intra et interbandes et devrait apporter des renseignements plus précis et complets sur la structure électronique du composé $\mathrm{MnAu}_{2}$.

\section{Bibliographie}

[1] Meyer, A. J. P. et Taglang, P., J. Physique 17 (1956) 457.

[2] Herpin, A. et Meriel, P., J. Physique 22 (1961) 337.

[3] Patterson, D. O., Thomson, J. O., Huray, P. G. and RoBerTs, L. D., Phys. Rev. B 2 (1970) 2440.

[4] JUng, C. et SToll, M. P. Opt. Commun. 21 (1977) 167.

[5] Johnson, P. B. and Christy, R. W., Phys. Rev. B 6 (1972) 4370.
[6] ThEYe, M. L., Phys. Rev. B 2 (1970) 3060.

[7] Winsemius, P., Thèse Leiden (Nederland) 1973.

[8] Lynam, P., Proctor, W., Puri, S. M. and Scurlock, R. G., Phys. Rev. B 2 (1970) 2448.

[9] Wells, P., J. Phys. F : Metal Phys. 4 (1974) 767. 